\title{
A 300-mV $\Delta \Sigma$ Modulator Using a Gain-Enhanced, Inverter-Based Amplifier for Medical Implant Devices
}

\author{
Ali Fazli Yeknami \\ School of Electrical Engineering and Computer Science, Oregon State University, \\ 1148 Kelley Engineering Center, Corvallis, OR 97331, USA; fazliyea@oregonstate.edu; \\ Tel.: +1-541-737-3617; Fax: +1-541-737-1300 \\ Academic Editor: Emre Salman \\ Received: 30 September 2015; Accepted: 8 March 2016; Published: 11 March 2016
}

\begin{abstract}
An ultra-low-voltage low-power switched-capacitor (SC) delta-sigma $(\Delta \Sigma)$ modulator running at a supply voltage as low as $300 \mathrm{mV}$ is presented for biomedical implant devices, e.g., cardiac pacemakers. To reduce the supply voltage, an inverter-based amplifier is used in the integrators, whose DC gain and gain-bandwidth (GBW) are boosted by a simple current-mirror output stage. The full input-feedforward loop topology offers low integrators internal swing, supporting ultra-low-voltage operation. To demonstrate the concept, a second-order loop topology was chosen. The entire modulator operates reliably against process, voltage and temperature (PVT) variations from a $300 \mathrm{mV} \pm 10 \%$ supply voltage only, while the switches are driven by a charge pump clock boosting scheme. Designed in a $65 \mathrm{~nm} \mathrm{CMOS} \mathrm{technology} \mathrm{and} \mathrm{clocked} \mathrm{at} 256 \mathrm{kHz}$, the simulation results show that the modulator can achieve a $64.4 \mathrm{~dB}$ signal-to-noise ratio (SNR) and a $60.7 \mathrm{~dB}$ signal-to-noise and distortion ratio (SNDR) over a $1.0 \mathrm{kHz}$ signal bandwidth while consuming $0.85 \mu \mathrm{W}$ of power.
\end{abstract}

Keywords: delta-sigma modulator; gain-enhanced inverter-based amplifier; biomedical implant devices; clock boosting; ultra-low voltage; low power

\section{Introduction}

Supply voltage reduction, as a direct consequence of the process technologies' scaling, is further enforced by battery-operated biomedical implant devices, such as pacemakers, cardiac defibrillators and neural recording integrated circuits, to make their operating supply compatible with human body potentials [1,2], in the range of few hundreds of $\mathrm{mVs}$. Therefore, designing analogue-to-digital converters (ADCs) operating at a very low supply voltage is inevitable for the measurement of various electrophysiological signals (e.g., ECGs, EEGs, etc.).

Pacemakers need to sense the cardiac signals, which mainly are situated in very low frequencies from nearly DC to several hundred hertz [3-7]. The cardiac signals are sensed by the low-noise amplifier, amplified by a gain stage; the undesired interferences are filtered out, and then, the detected analog signal is digitized by a back-end ADC. The ADC architecture and circuit design play a key role in maintaining ultra-low-power efficiency while providing a high conversion accuracy (or resolution). ADC resolutions from eight bit to 13 bit have been reported previously [5-7]. The low frequency noise, including flicker noise, needs to be treated properly in the circuit implementation.

There are two critical factors that can determine the lowest operating power supply of the traditional sigma-delta $(\Delta \Sigma)$ modulators: operational transconductance amplifiers (OTAs) in the integrators and the adequate driving voltage of the switching transistors [8-14]. The conventional analog circuit topologies are no longer practical in ultra-low-voltage operations (below $0.6 \mathrm{~V}$ ), and also, stacking more than two transistors is impossible due to the limited overdrive and voltage headroom. The inverter is the simplest amplifier, which can sustain a supply voltage of less than the sum of 
the absolute threshold voltages of the NMOS and PMOS transistors [12], also known as a Class-C inverter. The DC gain and gain-bandwidth product (GBW) of the CMOS inverter degrade significantly at supplies far below the nominal $\mathrm{V}_{\mathrm{TN}}+\left|\mathrm{V}_{\mathrm{TP}}\right|=0.62 \mathrm{~V}$ and need to be enhanced for a robust and high-performance modulator design. Chae and Han [12] proposed an inverter-based $\Delta \Sigma$ modulator using a cascode inverter for boosting the DC gain. In this paper, we present a fully-differential second-order feedforward $\Delta \Sigma$ modulator for ultra-low-voltage low-power biomedical applications, which uses a novel gain-enhanced inverter-based, current mirror amplifier to replace the OTA in the integrators. Moreover, a clock boosting scheme is used to sufficiently drive the switching transistors. While all transistors operate at gate voltage less than $300 \mathrm{mV}$, the effective gate voltage of the switches is $600 \mathrm{mV}$ with the aid of a charge pump clock doubler [13]. The single-bit quantizer, including the preamplifier, dynamic comparator and latch, was designed in a deep sub-threshold regime, providing very high power efficiency.

The rest of the paper is organized as follows: Section 2 describes the proposed gain-enhanced inverter-based amplifier and its advantages and drawbacks. Section 3 discusses the modulator architecture and its low-voltage and low-power design considerations. Section 4 presents the modulator circuit design. Section 5 discusses the simulation results. In Section 6, the proposed modulator is compared to the reported state-of-the-art ultra-low-voltage modulators. Conclusions are drawn in Section 7.

\section{Proposed Gain-Enhanced Inverter-Based OTA}

A novel inverter-based amplifier is proposed for ultra-low-voltage applications, which is composed of a Class-C inverter and a current mirror output stage. Figure 1 shows the schematics of the basic CMOS inverter, the cascode inverter and the proposed inverter-based, current mirror OTA (Figure 1c).

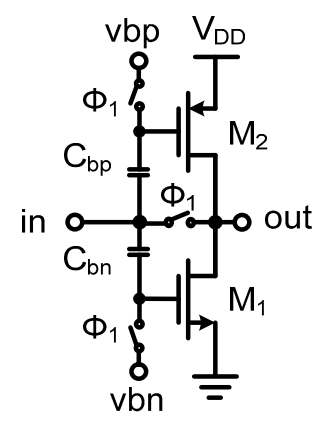

(a)

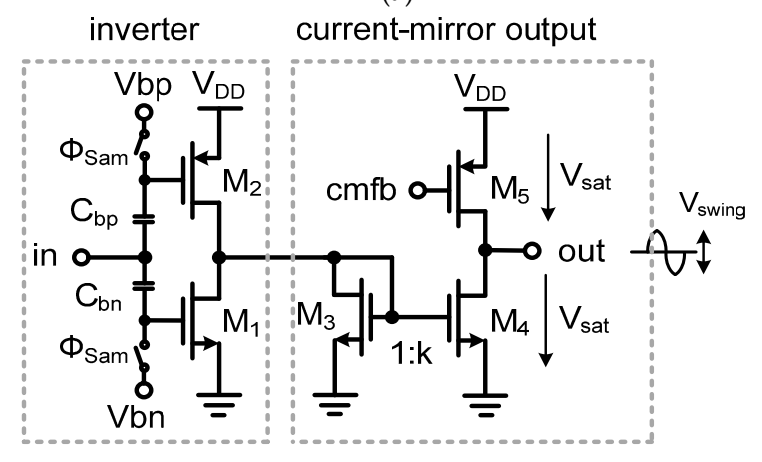

(c)

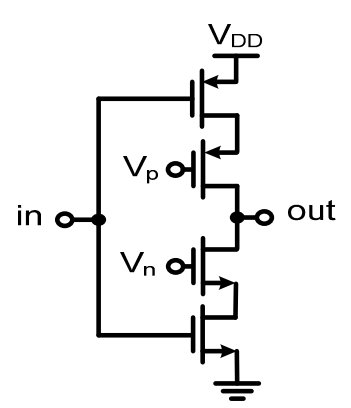

(b)

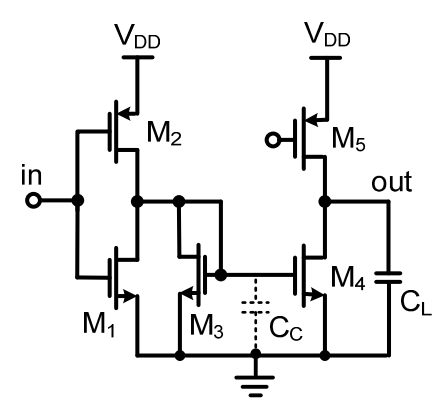

(d)

Figure 1. Schematic of the inverter amplifiers: (a) conventional CMOS inverter with biasing; (b) cascode inverter; (c) inverter-based current-mirror operational transconductance amplifier (OTA) using an input inverter stage and a current mirror output stage; single-ended is shown; (d) parasitic capacitance at internal node. 
A switched-capacitor (SC) biasing scheme using floating capacitors $C_{b p}$ and $C_{b n}$ (Figure 1c) is used to define the operating point of the inverter $\mathrm{M}_{1}-\mathrm{M}_{2}$. These capacitors are periodically refreshed by the bias voltages $V b n$ and $V b p$ during the sampling phase $\phi_{\text {Sam }}\left(\phi_{\text {Sam }}=\phi_{1 \mathrm{~d}}\right.$ in the first integrator; $\phi_{\text {Sam }}=\phi_{2 \mathrm{~d}}$ in the second integrator) and work as floating batteries between the input and the gates of the transistors. It is worth mentioning that the pre-charging $V b n$ and $V b p$ occurs simultaneously with sampling the signal onto $C_{S 1}\left(C_{S 2}\right)$ and the inverter offset onto $C_{C 1}\left(C_{C 2}\right)$ in phase $\phi_{\text {Sam }}$ (Figure 4). The sampling clock speed is low (i.e., $256 \mathrm{kHz}$ ), so initially, the $C_{b p}=C_{b n}=0.5 \mathrm{pF}$ are charged in the early phase of $\phi_{\text {Sam }}\left(C_{b p}=C_{b n}<<C_{C i}\right)$, and then, the amplifier is placed in the unity feedback configuration for offset sampling when its biasing was set appropriately. The $V b p$ and $V b n$ are generated using a constant $-g_{m}$ biasing circuit followed by a level shifter to eliminate the $V_{\text {sat }}$ problem as in [15], which provides wider overdrive voltage and, in turn, a smaller transistor width. At the output stage, an energy-efficient SC common-mode feedback (CMFB) circuit is employed [8], which derives the gate of PMOS transistor $\mathrm{M}_{5}$, in order to set the output $\mathrm{CM}$ level at the middle of the supply voltage for the maximum output swing.

The DC gain and GBW of the CMOS inverter degrade significantly at supplies far below the nominal $\mathrm{V}_{\mathrm{TN}}+\left|\mathrm{V}_{\mathrm{TP}}\right|$, which need to be enhanced for a robust and high-performance modulator design. The aim of the proposed technique is to boost the gain and GBW simultaneously by mirroring a small fraction ( $\sim 10 \%$ ) of the bias current of the inverter $\mathrm{M}_{1}-\mathrm{M}_{2}$ through transistor $\mathrm{M}_{3}$ to the output. The factor $k$ is the current ratio of the current mirror $\mathrm{M}_{3}-\mathrm{M}_{4}$ and is defined as $(\mathrm{W} / \mathrm{L})_{\mathrm{M} 4} /(\mathrm{W} / \mathrm{L})_{\mathrm{M} 3}$. The approximate gain and GBW of the proposed amplifier shown in Figure 1c can be determined from its small-signal model using sub-threshold current as:

$$
\begin{gathered}
A_{e n}=\frac{g_{m 1}+g_{m 2}}{g_{m 3}+g_{d s 1}+g_{d s 2}+g_{d s 3}} \cdot \frac{g_{m 4}}{g_{d s 4}+g_{d s 5}} \\
\cong\left(g_{m 1}+g_{m 2}\right) \times k \times R_{\text {out }} \\
G B W=\frac{k \times\left(g_{m 1}+g_{m 2}\right)}{2 \pi C_{L}}=\frac{k(2-\alpha) I_{D 2}}{2 \pi C_{L} n V_{T}}
\end{gathered}
$$

where $g_{m i}$ and $g_{d s i}$ represent the transconductance and output conductance of the $i$-th transistor, respectively. $R_{\text {out }}$ is the total output resistance, which is equal to $\left(g_{d s 4}+g_{d s 5}\right)^{-1}$. $C_{L}$ is the total load capacitor for the frequency compensation. $n$ and $V_{T}$ are the sub-threshold parameters. We consider $\alpha=I_{D 3} / I_{D 2}$ as the ratio of the currents of $\mathrm{M}_{3}$ and $\mathrm{M}_{2}$. In addition, it can be shown that the term $g_{m 4} /\left(g_{m 3}+g_{d s 1}+g_{d s 2}+g_{d s 3}\right)$ is approximately equal to $k$. A large device size of $\mathbf{M}_{1}-\mathbf{M}_{2}$ has to be prevented, as it creates large parasitic capacitors, which can limit the amplifier speed.

To explain the gain-enhancement technique, the DC gain of the basic inverter (Figure 1a) and the proposed amplifier (Figure 1c) given by Equation (1) can be written in the following forms in Equations (3) and (6):

$$
A_{0}=\left(g_{m 1}+g_{m 2}\right) R_{\text {out }}=\frac{2 I_{D}}{n V_{T}} \times \frac{1}{\lambda I_{D}}=\frac{2}{n \lambda V_{T}}
$$

$V_{T}$ is the thermal voltage; $n$ is the non-ideality factor; and $\lambda$ is the channel length modulation coefficient. $A_{0}$ is the intrinsic gain achieved by an inverter in the sub-threshold regime. In deep submicron technologies, by shrinking the transistor length, the effect of channel length modulation becomes more important. As a consequence, the output resistance and, thus, the DC gain of the inverter are normally low.

In Figure 1c, the bias current of the diode-connected device (i.e., $I_{D 3}$ ) is equal to $\alpha I_{D 2}$, where $\alpha$ in this design is approximately 0.1. The $M_{1}$ transistor then shunts the rest of the current to the ground. Assuming $\mathrm{M}_{1}$ carries $(1-\boldsymbol{\alpha}) \times I_{D 2}$, from Equation (1), the gain of the proposed OTA can be expressed as:

$$
A_{e n}=\left(g_{m 1}+g_{m 2}\right) \times k \times R_{o u t}=\left[\frac{(1-\alpha) I_{D 2}}{n V_{T}}+\frac{I_{D 2}}{n V_{T}}\right] \times k \times \frac{1}{\lambda_{4} I_{D 4}}
$$


Now, the biasing current of $\mathrm{M}_{4}$ is:

$$
I_{D 4}=k \times I_{D 3}=k \times \alpha I_{D 2}
$$

Finally, the enhanced gain of the OTA is given by:

$$
A_{e n}=\frac{2-\alpha}{2 \alpha} \times \frac{2}{n \lambda V_{T}}=\left(\frac{1}{\alpha}-0.5\right) A_{0}
$$

From Equation (6), it can be seen that the gain is enhanced $(1 / \alpha-0.5)$-times. The gain increase can be adjusted by the $\alpha$ factor during the design. With $\alpha=0.1$ in this design, the gain can be enhanced 9.5-times directly from Equation (6). With $\alpha=I_{D 3} / I_{D 2}$ as the ratio of the currents in $\mathrm{M}_{3}$ and $\mathrm{M}_{2}$, the current mismatch can affect the gain enhancement (Section 2.2). In typical corner simulation from a 0.3 $V$ supply, the $\alpha$ factor is about 0.12 , which corresponds to a gain enhancement of eight-times, whereas in the worst-case corner, it is approximately 0.13 , corresponding to a gain enhancement of seven-times.

Figure 2a shows the variations of the DC gain with respect to the supply voltage $\left(V_{D D}\right)$ for the basic inverter shown in Figure 1a and the gain-enhanced inverter shown in Figure 1c. Figure 2b,c also shows the variations of the GBW as a function of the $V_{D D}$ for both the basic inverter and the gain-enhanced inverter. For PMOS transistor $\mathrm{M}_{2}$, the overdrive voltage (i.e., $V_{D D}-V b p-\left|V_{T P}\right|$ ) depends on $V_{D D}$ and increases by increasing $V_{D D}$. Similarly, for NMOS device $\mathrm{M}_{1}$, the overdrive voltage (i.e., $V b n-V_{T N}$ ) is enhanced linearly with $V_{D D}$ to accommodate a wider input linear range in this analysis.

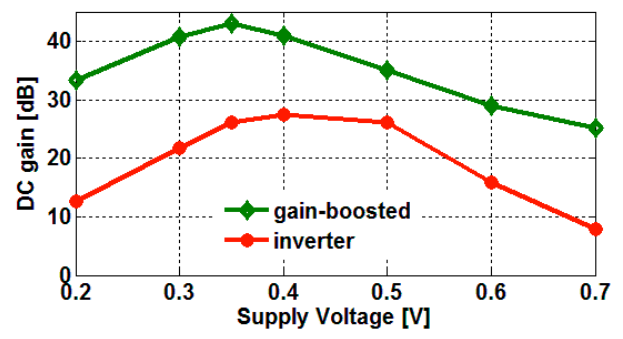

(a)

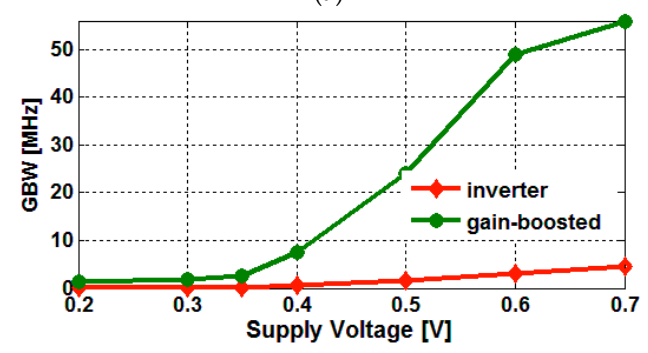

(b)

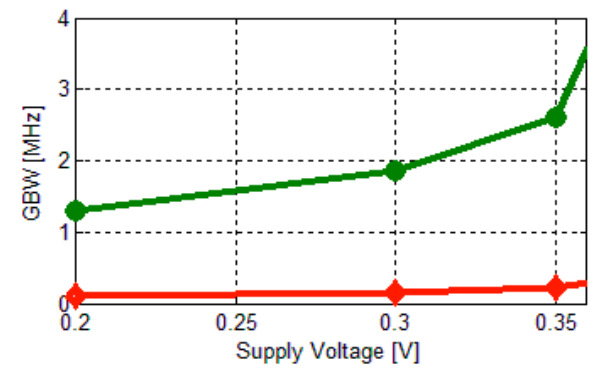

(c)

Figure 2. (a) DC gain and (b) gain-bandwidth (GBW) variations versus supply voltage for the CMOS inverter shown in Figure 1a and the proposed gain-enhanced, inverter-based amplifier shown in Figure 1c; (c) the zoomed version of the Figure $2 b$ for clarity. 
For $V_{D D}$ s far below $V_{T N}+\left|V_{T P}\right|$, the DC gain and GBW degrade significantly. A large device size in $\mathrm{M}_{1}-\mathrm{M}_{2}$ has to be avoided, as this creates large parasitic capacitances, which can limit the OTA performance. The aim of this work is to enhance the gain and GBW simultaneously by mirroring a small fraction of the bias current of the inverter $\mathrm{M}_{1}-\mathrm{M}_{2}$ to the output stage (with $\alpha \sim 10 \%$ and $k=8$ ).

Shown in Figure 2, with the current mirror gain-enhancement technique, the simulated DC gain and GBW increase to $40 \mathrm{~dB}$ and $1.9 \mathrm{MHz}$ from $22 \mathrm{~dB}$ and $0.38 \mathrm{MHz}$, respectively, from a $0.3 \mathrm{~V}$ supply and a $3 \mathrm{pF}$ load capacitance in a typical (TT) process corner, at $27^{\circ} \mathrm{C}$. The phase margin is $66^{\circ} \mathrm{C}$. The transistors sizes of the developed inverter-based current mirror amplifier are summarized in Table 1. The DC gain gets worse in FS (fast NMOS, slow PMOS) and SF (slow NMOS, fast PMOS) process corners (i.e., $37 \mathrm{~dB}$ at $V_{D D}=0.3 \mathrm{~V}$ ), whereas its deviation is trivial for $\mathrm{FF}$ and $\mathrm{SS}$ corners (i.e., $39 \mathrm{~dB}$ at $\left.V_{D D}=0.3 \mathrm{~V}\right)$.

Table 1. Transistor aspect ratios and bias setting for the gain-enhanced current mirror inverter amplifier in a $65 \mathrm{~nm}$ CMOS.

\begin{tabular}{cc}
\hline Transistor & W/L $(\mu \mathrm{m} / \mu \mathrm{m})$ \\
\hline $\mathrm{M}_{1}$ & $1 / 0.8$ \\
$\mathrm{M}_{2}$ & $9 / 0.8$ \\
$\mathrm{M}_{3}$ & $2 / 0.2$ \\
$\mathrm{M}_{4}$ & $16 / 0.2$ \\
$\mathrm{M}_{5}$ & $5 / 0.25$ \\
\hline Capacitor & Value (pF) \\
\hline $\mathrm{C}_{\text {bn }}=\mathrm{C}_{\text {bp }}$ & 0.5 \\
$\mathrm{C}_{\text {Load }}$ & 3.0 \\
\hline
\end{tabular}

In practice, the gain enhancement can be restricted by several factors: phase margin, matching of bias currents between the $\mathrm{M}_{1}$ and $\mathrm{M}_{3}$ transistors and thermal noise. These factors are discussed below.

\subsection{Frequency Response and Internal Parasitic Pole}

Using the gain-enhancement technique increases the impedance of the internal node at the gate of the diode-connected $\mathrm{M}_{3}$. The total parasitic capacitance at this node is represented by $C_{P}$ in Figure $1 \mathrm{~d}$. The parasitic non-dominant pole due to the impedance $1 / g_{m 3}$ of the transistor $\mathrm{M}_{3}$ operating in the sub-threshold regime and the parasitic capacitor $C_{P}$ can be expressed as:

$$
P_{n d}=\frac{g_{m 3}}{2 \pi C_{P}}=\frac{\alpha I_{D 2}}{2 \pi C_{P} n V_{T}}
$$

where $C_{P}$ is approximately $C_{g s 3}+C_{g s 4}$. To maintain a reasonably safe phase margin, the $P_{n d}$ has to be placed more than three-times the unity GBW given by Equation (2). Thus, the following criteria for $\alpha$ can be derived with respect to $C_{P} / C_{L}$ and $k$ :

$$
\frac{1}{\alpha}-0.5 \leqslant \frac{1}{6 k} \frac{C_{L}}{C_{P}}
$$

Recalling $(1 / \boldsymbol{\alpha}-0.5)$ in Equation (6) as the gain-enhancement factor, Equation (8) represents the maximum gain-enhancement that can be achieved by the proposed technique. The larger the $C_{L} / C_{P}$, the greater is the gain enhancement and the phase margin, but this costs more power. The dominant pole in this design is set by $C_{L}$, which can be expressed by:

$$
P_{1}=\frac{1}{2 \pi R_{\text {out }} C_{L}}=\frac{g_{d s 4}+g_{d s 5}}{2 \pi C_{L}}
$$




\subsection{Bias Current Matching}

As described earlier, the ratio of the bias currents of transistors $\mathrm{M}_{3}$ and $\mathrm{M}_{2}$, defined as $\alpha=I_{D 3} / I_{D 2}$, plays an important role for enhancing the amplifier gain given by Equation (6). Practically, the matching between $I_{D 3}=\alpha I_{D 2}$ and $I_{D 1}=(1-\alpha) I_{D 2}$ determines the real $\alpha$ factor and, thus, the gain enhancement. To ensure good matching between those currents, the gate bias voltages of $M_{1}-M_{2}$ both are generated from the same reference current source using current mirrors. For good matching, transistors should be sized properly.

\subsection{Thermal Noise}

A drawback of the gain-enhanced, inverter-based amplifier, as compared to the basic CMOS inverter, is that it exhibits more input-referred thermal noise, related to the $g_{m 3}$ of the device $\mathrm{M}_{3}$. Since the correlated double-sampling (CDS) technique, as an auto-zeroing technique, is used in the corresponding integrator, the low frequency flicker noise is attenuated at the cost of an increased white noise floor due to the noise folding accompanied by the sampling [16]. The foldover thermal noise of the integrator is the dominant source of the noise, in which the thermal noise is amplified by a factor GBW $/ f_{S}$, with sampling frequency $f_{S}[16]$. As a result, the input-referred noise power of the Class-C inverter and the gain-enhanced inverter shown in Figure 1c, denoted by GE-inv, can be expressed, respectively, as:

$$
\begin{gathered}
\overline{V_{n-f o l d, i n v}^{2}}=\frac{G B W}{f_{s}} \cdot \frac{4 k T \gamma}{g_{m 1}+g_{m 2}}\left[v^{2} / H z\right] \\
\overline{V_{n-f o l d, G E-i n v}^{2}}=\frac{G B W}{f_{s}} \cdot \frac{4 k T \gamma}{g_{m 1}+g_{m 2}}\left(1+\frac{g_{m 3}}{g_{m 1}+g_{m 2}}\right)\left[v^{2} / H z\right]
\end{gathered}
$$

where the noise of the gain-enhanced amplifier has an additional term corresponding to $g_{m 3}$. Therefore, $\mathrm{M}_{3}$ has to be sized carefully, such that the thermal noise is minimized and the non-dominant pole, i.e., $g_{m 3} / 2 \pi C_{P}$ with $C_{P}$ the parasitic capacitance at the gate of $\mathrm{M}_{3}$ and $\mathrm{M}_{4}$, is placed more than $3 \times$ the GBW for a reasonably safe phase margin.

\section{Modulator Architecture}

Output swing is of great importance in ultra-low-voltage low-power designs, which directly determines the modulator dynamic range (DR) and, ultimately, the power consumption. The minimum swing $V_{\text {swing }}$ that the amplifier shown in Figure 1c can still operate with imposes a hard limit equal to $2 V_{\text {sat }}+V_{\text {swing }}$ for the supply voltage scaling, with saturation voltage $V_{\text {sat }}$. The limited voltage swing is therefore translated into the demanding requirement of a low-swing loop topology. Compared to the traditional feedback topology, the full input-feedforward architecture suggests the integrators to process only the quantization error, thereby reducing the integrators' swing considerably [17]. This is beneficial for the amplifiers' relaxed requirements for slew-rate. Figure 3a shows the input-feedforward loop architecture for a second-order modulator. Half-cycle delay integrators are adopted in this structure to realize the CDS scheme for the inverter's offset cancellation. The loop coefficients were optimized with behavioral simulations as $\left(a_{1} a_{2} c_{1} c_{2} c_{3}\right)=(0.10 .6171)$. The coefficients are determined from the loop stability constraint, the maximum linear swing of the integrators and the required SNR. Figure $3 \mathrm{~b}$ depicts the magnitude of the signal transfer function (STF) and the noise transfer function (NTF) of the target modulator. As expected, the STF is unity, and the NTF has a $40 \mathrm{~dB} / \mathrm{dec}$ noise suppression in the baseband. 


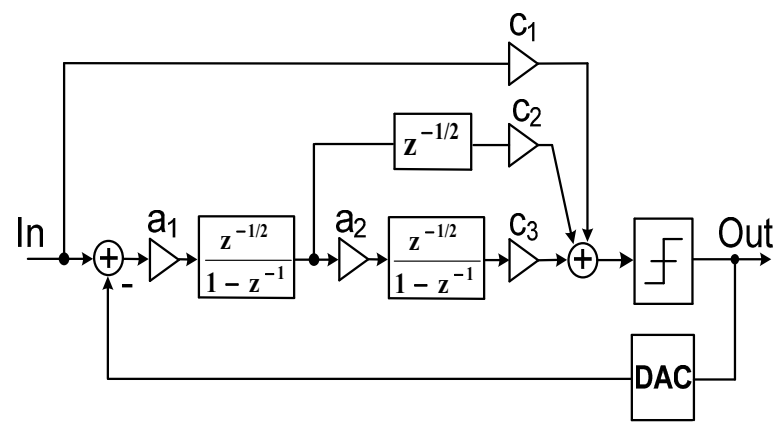

(a)

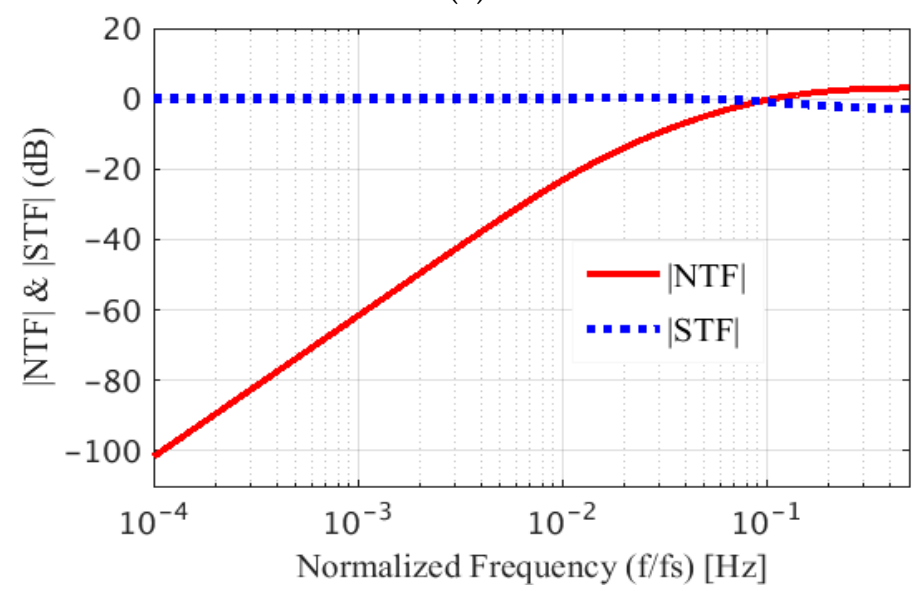

(b)

Figure 3. (a) The scaled modulator input-feedforward architecture using a second-order loop filter and a single-bit quantizer. Integrators with half-cycle delay were used to adopt the built-in correlated double-sampling (CDS) scheme for offset cancellation and 1/f noise attenuation; (b) the magnitude of the noise transfer function (NTF) and signal transfer function (STF) of the modulator with respect to the normalized frequency.

The single-bit quantizer is inherently linear. A multi-bit quantizer is not preferable for the low power and moderate resolution in this application, because the internal DAC becomes nonlinear, requiring dynamic element matching (DEM) or other complementary techniques for DAC linearization, which increase the hardware complexity and, thus, the total power consumption.

\section{Modulator Circuit Design and Building Blocks}

In this section, the details of modulator circuit design and its building blocks are discussed.

\subsection{Modulator Circuit}

Figure 4 shows the schematic of the designed second-order SC $\Delta \Sigma$ modulator using the proposed pseudo-differential integrators. The SC CMFB circuit is not shown and is omitted for simplicity. The proposed inverter-based amplifier in feedback configuration does not provide a virtual ground at the integrator input, because it has only one input terminal (Figure 1c). Combined with the CDS technique to cancel out the input offset and to attenuate the $1 / \mathrm{f}$ noise [16], two instances of the designed inverter-based amplifier are used to realize a pseudo-differential integrator with a virtual ground. There is a half-cycle delay between the integrators, i.e., when the first integrator samples the input onto $C_{S 1}$ and the offset of the inverter onto $C_{C 1}$, the second integrator is in charge transfer phase and vice versa. Since all feedforward paths have to create full delay paths according to Figure $3 a$, a half-cycle delay element is inserted in the internal feedforward path using SC implementation. 


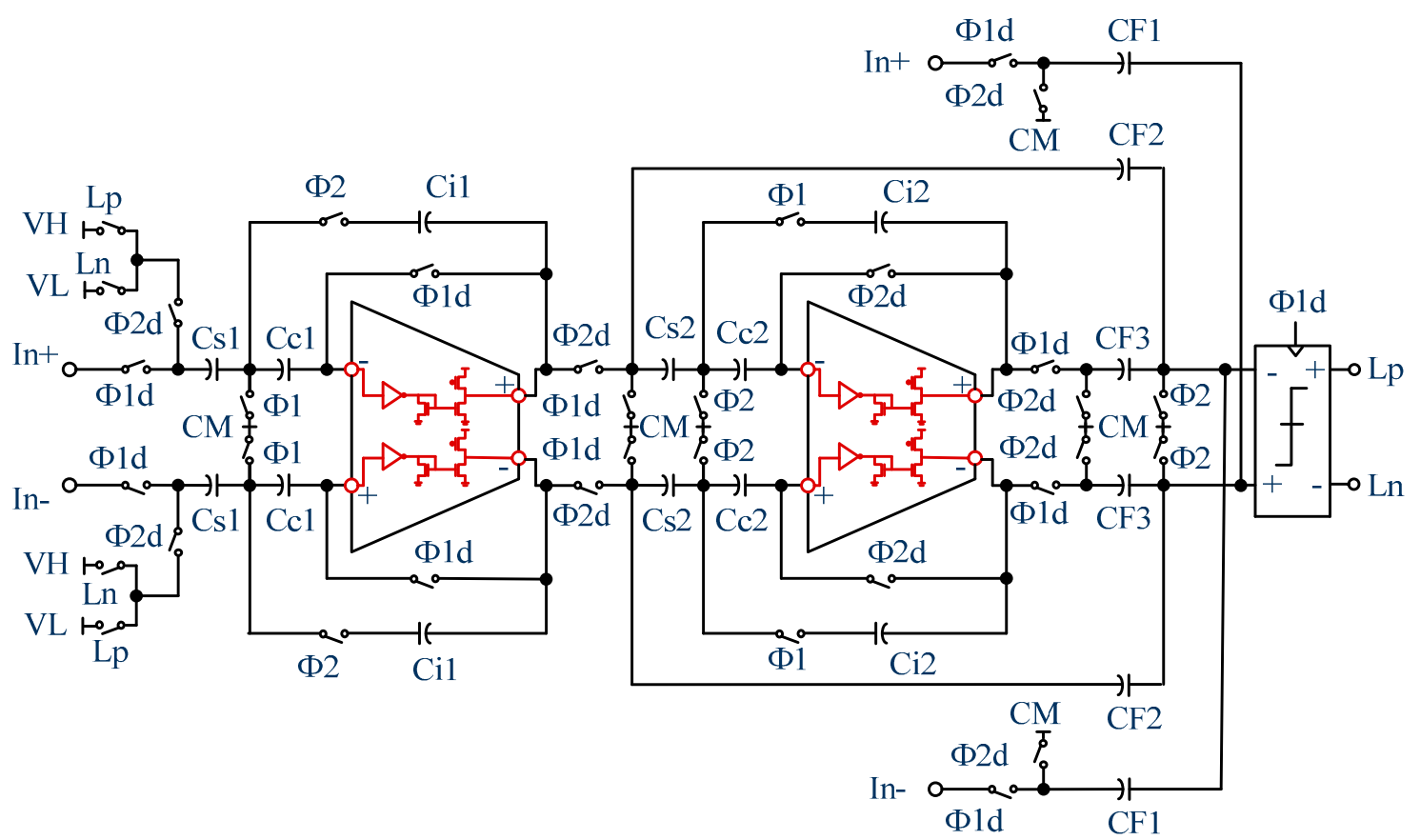

Figure 4. Schematic diagram of the one-bit second-order switched-capacitor (SC) delta-sigma modulator using pseudo-differential integrators. Each integrator circuit shown in red represents the proposed inverter-based current mirror amplifier discussed in Section 2. VH and VL denote the positive and negative reference voltages. $\mathrm{CM}$, common mode.

The summation of the feedforward paths is realized by the parallel capacitor branches at the quantizer input by using an SC passive network. The feedforward architecture is preferable due to its relaxed OTA's performance requirements and low internal swings.

\subsection{Low-Voltage Clocking and Clock-Boosting Circuitries}

Figure 5 shows the non-overlapping clock generation circuitry used to provide the clock timing required by the modulator. The entire circuit employs low- $V_{T H}$ low-power (LVTLP) devices enabling operation at $300 \mathrm{mV}$. The transistor aspect ratios of the CMOS inverters and the output buffers are specified in $\mu \mathrm{m} / \mu \mathrm{m}$ in the figure. The aspect ratios of the CMOS NAND gate for PMOS and NMOS devices are $0.24 \mu \mathrm{m} / 1.5 \mu \mathrm{m}$ and $0.135 \mu \mathrm{m} / 1.5 \mu \mathrm{m}$, respectively. In order to open and close the switches properly, they are implemented as transmission gates with low- $V_{T H}$ transistors, driven by an ultra-low-voltage charge-pump clock doubler [13].

Figure 6 shows the schematic of the clock boosting circuit, which is composed of a charge-pump voltage doubler with two cross-coupled NMOS devices, two capacitors as large as $5 \mathrm{pF}$ and two inverters as the buffer and level shifter. Low- $V_{T H}$ devices were employed in the charge-pump circuit and level shifters for low voltage operation at $300 \mathrm{mV}$. The $\phi 1 \mathrm{~L}$ and $\phi 2 L$ are two non-overlapping clocks generated by the circuit in Figure 5 with a voltage level equal to $0.3 \mathrm{~V}$, whereas $\phi 1 \mathrm{H}$ and $\phi 2 \mathrm{H}$ are level shifted up to $0.6 \mathrm{~V}$. Hereafter, for simplicity, we denote $\phi 1 \mathrm{H}$ and $\phi 2 \mathrm{H}$ as $\phi 1$ and $\phi 2$, as in Figure 4 . In a similar way, the $\phi 1 d$ and $\phi 2 d$ are generated and level shifted. 


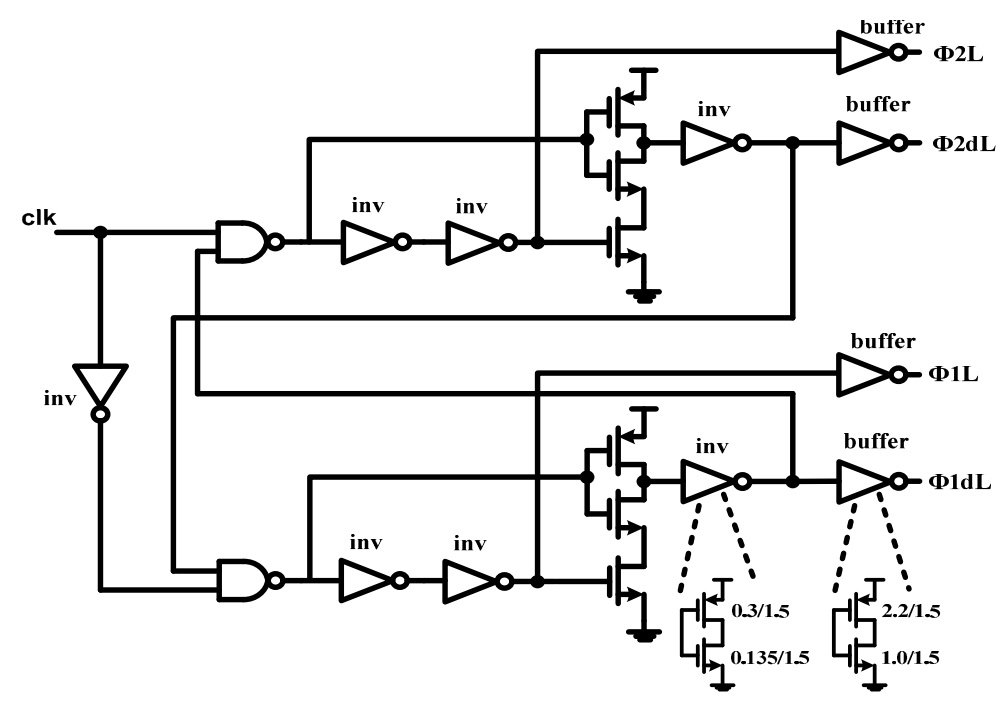

Figure 5. Clock generation circuitry using low- $V_{T H}$ low-power (LVTLP) devices. The transistor aspect ratios of the inverters and the output buffers are given in $\mu \mathrm{m} / \mu \mathrm{m}$. This provides two non-overlapping clocks $(\phi 1 L, \phi 2 L)$ and their delays $(\phi 1 d L, \phi 2 d L)$ to avoid signal-dependent charge injection.

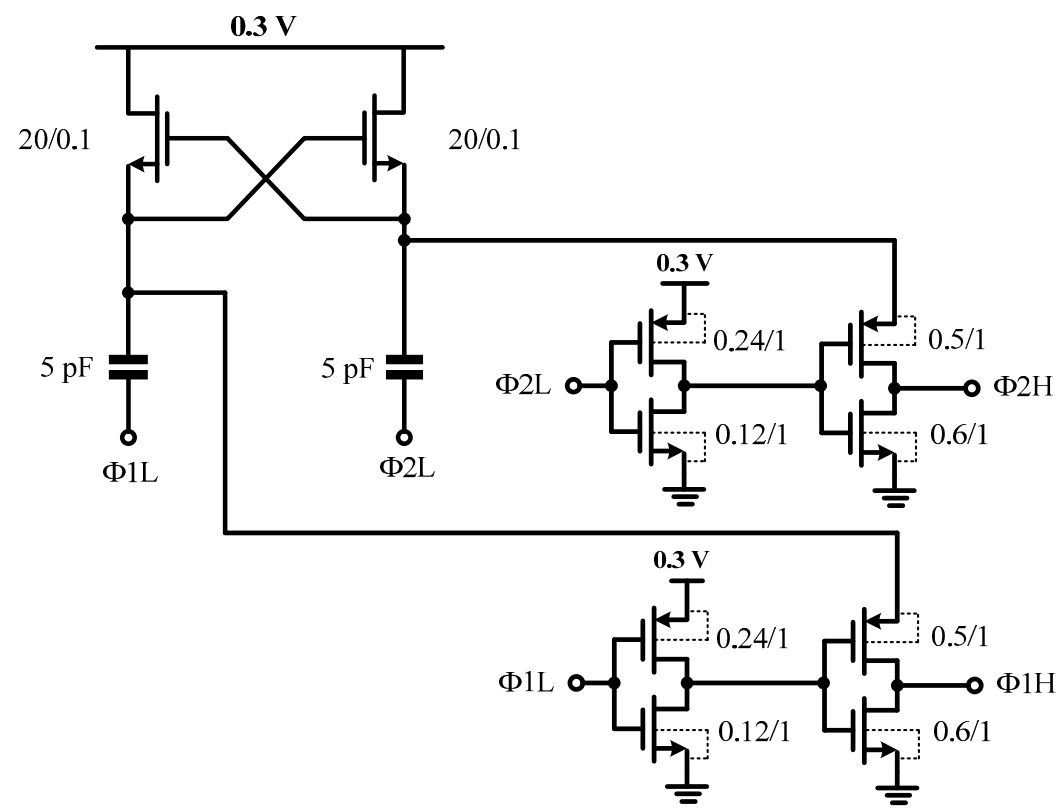

Figure 6. Clock doubler with a charge-pump circuit and level shifters using low- $V_{T H}$ devices. The transistor aspect ratios are specified in $\mu \mathrm{m} / \mu \mathrm{m}$.

\subsection{Ultra-Low-Voltage Sub-Threshold Quantizer}

The single-bit quantizer is implemented using a dynamic comparator preceded by a single-stage preamplifier, as shown in Figure 7. Table 2 summarizes the transistors dimension in $\mu \mathrm{m}$. At a $0.3 \mathrm{~V}$ supply, the regular preamplifier and comparator circuits do not function properly in the strong inversion. 


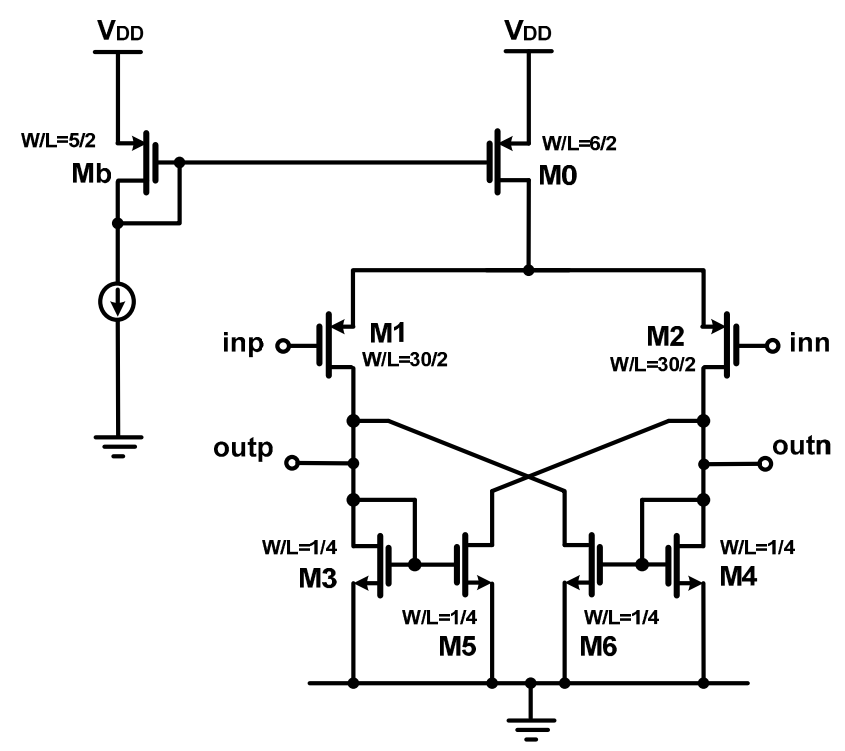

(a)

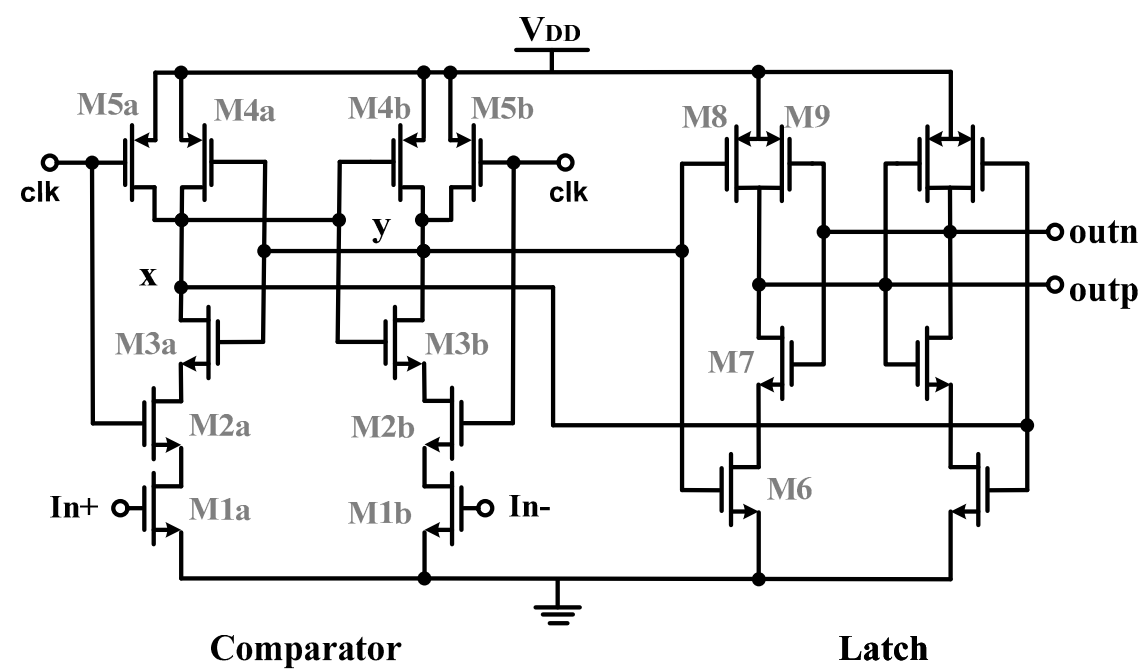

(b)

Figure 7. (a) Low-voltage differential gain-enhanced preamplifier circuit operating in the sub-threshold regime. The transistors aspect ratios are specified in $\mu \mathrm{m} / \mu \mathrm{m}$. (b) Low-voltage dynamic comparator and latch designed in the sub-threshold regime. The transistors aspect ratios are summarized in Table 2.

Table 2. Transistor aspect ratios of the clocked comparator and latch designed in the sub-threshold regime.

\begin{tabular}{cc}
\hline Transistors in Comparator & W/L $(\mu \mathbf{m} / \mu \mathbf{m})$ \\
\hline $\mathrm{M}_{1 \mathrm{a}} / \mathrm{M}_{1 \mathrm{~b}}$ & $8 / 0.18$ \\
$\mathrm{M}_{2 \mathrm{a}} / \mathrm{M}_{2 \mathrm{~b}}$ & $4 / 0.18$ \\
$\mathrm{M}_{3 \mathrm{a}} / \mathrm{M}_{3 \mathrm{~b}}$ & $3 / 0.18$ \\
$\mathrm{M}_{4 \mathrm{a}} / \mathrm{M}_{4 \mathrm{~b}}$ & $25 / 0.18$ \\
$\mathrm{M}_{5 \mathrm{a}} / \mathrm{M}_{5 \mathrm{~b}}$ & $30 / 0.18$ \\
\hline Transistors in Latch & $\mathrm{W} / \mathrm{L}(\mu \mathrm{m} / \mu \mathrm{m})$ \\
\hline $\mathrm{M}_{6}$ & $1 / 0.18$ \\
$\mathrm{M}_{7}$ & $0.5 / 0.18$ \\
$\mathrm{M}_{8}$ & $1 / 0.18$ \\
$\mathrm{M}_{9}$ & $1 / 0.18$ \\
\hline
\end{tabular}


The input signals have a common-mode level equal to $0.15 \mathrm{~V}$ (i.e., $\left.V_{D D} / 2\right)$, which is not adequate to turn on the input transistors. The threshold voltage of the used low- $V_{T H}$ transistors in this technology itself is more than $0.15 \mathrm{~V}$. On the other hand, stacking more than three transistors is impractical. Therefore, the single-bit quantizer (including the preamplifier circuit and the dynamic comparator and latch) is designed in the deep sub-threshold regime, providing very high power efficiency. The entire circuit dissipates only $6 \mathrm{nW}$, while clocked at $256 \mathrm{kHz}$. The quantizer operating at $0.3 \mathrm{~V} \pm 10 \%$ was simulated against process, voltage and temperature (PVT) variations. It is capable of detecting an input signal as low as $200 \mu \mathrm{V}$, whereas the modulator's least significant bit (LSB) is $300 \mu \mathrm{V}$ for a full-scale reference voltage of $300 \mathrm{mV}$ and 10 bit resolution.

The gain of the preamplifier degrades to $5 \mathrm{~dB}$ at a $0.3 \mathrm{~V}$ supply. Thus, it employs a gain-enhanced positive feedback in order to boost the low-frequency gain [2]. The preamplifier exploits low- $V_{T H}$ transistors, providing more headroom for ultra-low voltage operation. For robust function against PVT variations, the effect of mismatch and process variations over DC gain was simulated using Monte Carlo analysis. Figure 8a shows the histogram of the DC gain for 5000 runs, which demonstrates the mean value of $10.55 \mathrm{~dB}$. The frequency response was also simulated for 1000 runs, as depicted in Figure $8 \mathrm{~b}$. The worst-case DC gain in this plot is more than $9 \mathrm{~dB}$.

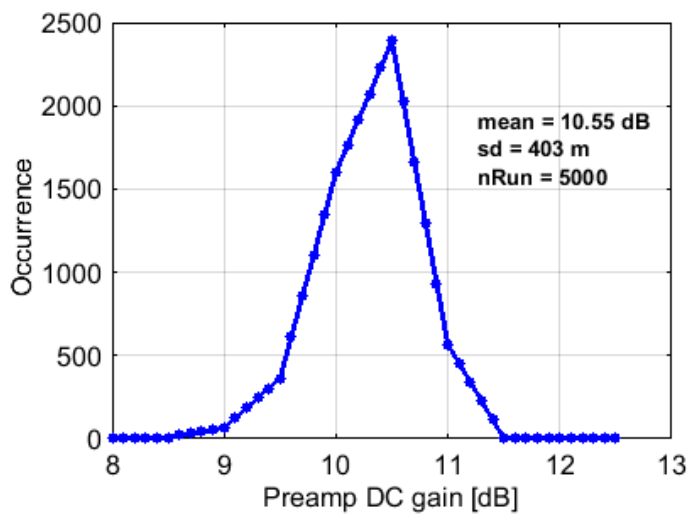

(a)

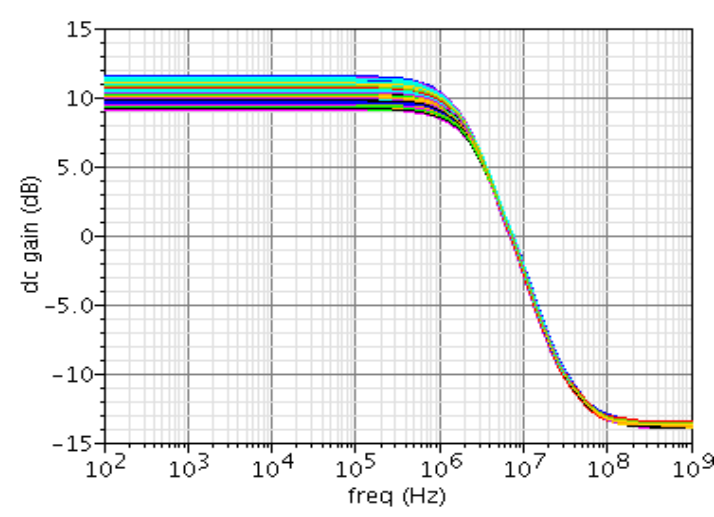

(b)

Figure 8. (a) Histogram of the DC gain of the low-voltage preamplifier shown in Figure 7a operating in the sub-threshold regime from the Monte Carlo simulations; (b) Bode plot of the frequency response from Monte Carlo simulations.

\section{Simulation Results}

The proposed $0.3 \mathrm{~V}$ delta-sigma modulator was designed and simulated in a $65 \mathrm{~nm}$ CMOS process. Figure 9 shows the output power spectrum for a $-2 \mathrm{~dB}$ full-scale (dBFS), $226 \mathrm{~Hz}$ sine-wave input. Figure 10 shows the power spectrum for a $960 \mathrm{~Hz}$ input, near the signal bandwidth edge, with a full scale input amplitude (i.e., $0 \mathrm{dBFS}$ ). The differential input signal range is $500 \mathrm{mV}_{\mathrm{pp}}$. The modulator performance is simulated against process corners and $10 \%$ supply voltage variations, the achieved worst-case peak SNR and SNDR are $64.4 \mathrm{~dB}$ and $60.7 \mathrm{~dB}$, respectively, within a $1.0 \mathrm{kHz}$ signal bandwidth with a $256 \mathrm{kHz}$ sampling clock frequency. The total power consumption from a $0.3 \mathrm{~V}$ power supply is $0.85 \mu \mathrm{W}$, in which the digital power is only $9 \%$, including clock generation and clock boosting circuitries. Figure 11 presents the modulator SNDR versus the input differential amplitude in dBFS. The performance metrics are summarized in Table 3. The resulting figure of merit (FOM) is $0.46 \mathrm{pJ} /$ conversion-step, by calculating $\mathrm{FOM}=$ power $/\left(2^{\mathrm{ENOB}} \times 2 \times\right.$ bandwidth $)$ with $\mathrm{ENOB}$ as the effective number of bits. The modulator can work up to a $0.5 \mathrm{~V}$ power supply. 


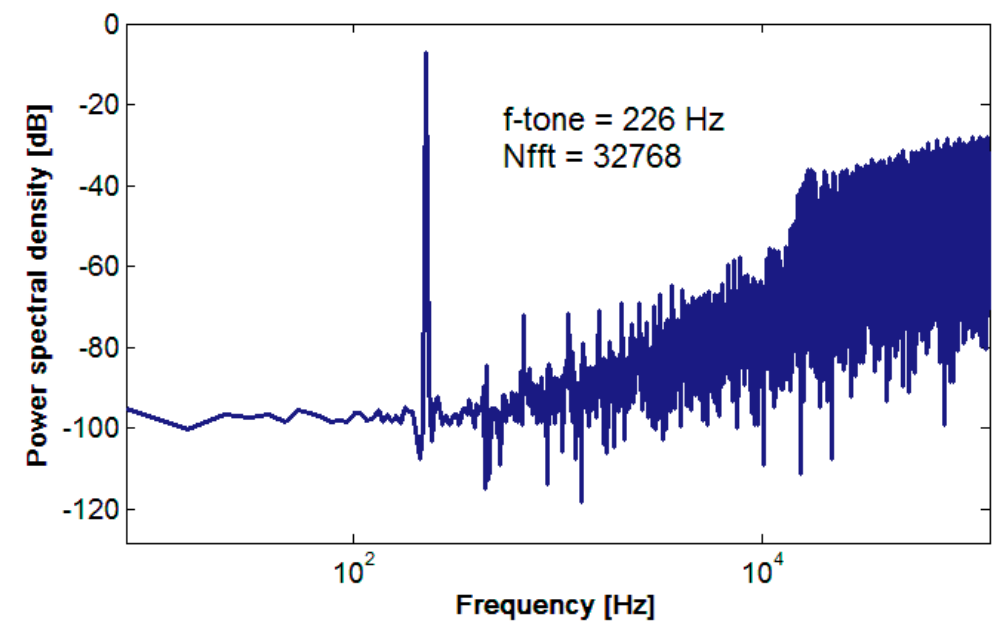

Figure 9. Simulated output power spectrum of the designed modulator for the input signal frequency of $226 \mathrm{~Hz}$ with a $-2 \mathrm{dBFS}$ amplitude.

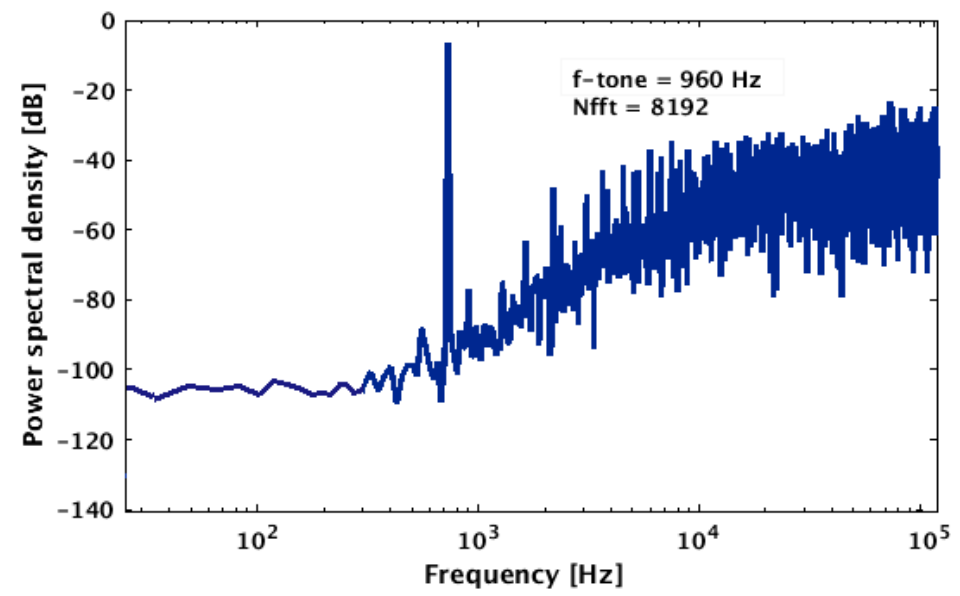

Figure 10. Simulated output power spectrum of the designed modulator for the input signal frequency of $960 \mathrm{~Hz}$ and a full-scale amplitude (0 dBFS). The effective signal bandwidth is $1 \mathrm{kHz}$.

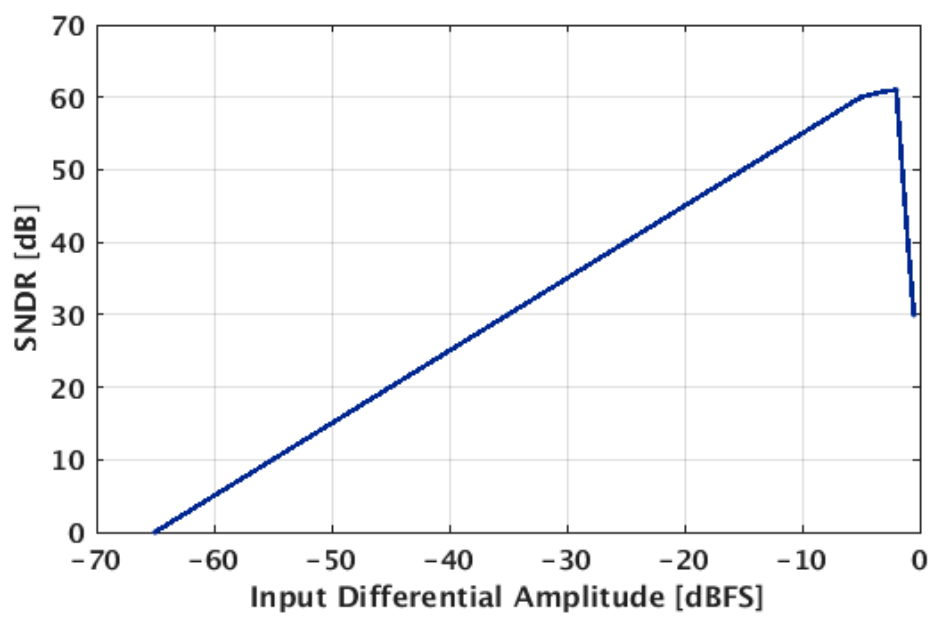

Figure 11. Simulated signal-to-noise and distortion ratio (SNDR) versus input differential amplitude in $\mathrm{dBFS}$. 
Table 3. Simulated performance results.

\begin{tabular}{cc}
\hline Metrics & Simulated Value \\
\hline Supply voltage & $0.3 \mathrm{~V}$ \\
Technology & $65 \mathrm{~nm} \mathrm{CMOS}$ \\
Clock frequency & $256 \mathrm{kHz}$ \\
Signal bandwidth & $1 \mathrm{kHz}$ \\
Differential input range & $0.5 \mathrm{~V}_{\text {peak- }}$ to - peak \\
Temperature range & 0 to $100{ }^{\circ} \mathrm{C}$ \\
\hline & slow corner $64.4 \mathrm{~dB}$ \\
Peak SNR & typical corner $65.5 \mathrm{~dB}$ \\
& fast corner $67 \mathrm{~dB}$ \\
\hline & slow corner $60.7 \mathrm{~dB}$ \\
Peak SNDR & typical corner $62 \mathrm{~dB}$ \\
& fast corner $63.2 \mathrm{~dB}$ \\
\hline Dynamic range & $65 \mathrm{~dB}$ \\
Power consumption & $0.85 \mu \mathrm{W}$ \\
\hline
\end{tabular}

\section{Comparison of the Power Efficiency}

The performance of the presented modulator in Sections 4 and 5 is compared to previous state-of-the-art low-voltage modulators in Table 4. Two commonly-used FOMs are employed, which are defined below:

$$
\begin{gathered}
\text { FOM }_{1}=\frac{\text { Power }}{2^{(S N D R-1.76) / 6.02} \times 2 \times B W} \\
\text { FOM }_{2}=D R_{d B}+10 \log \left(\frac{B W}{\text { Power }}\right)
\end{gathered}
$$

Table 4. Performance comparison with previously-reported low-voltage low-power delta-sigma modulators. FOM, figure of merit.

\begin{tabular}{ccccccc}
\hline Parameters & {$[15]$} & {$[18]$} & {$[19]$} & [9] $^{\dagger}$ & [20] & This Work $^{\dagger}$ \\
\hline$V_{D D}(\mathrm{~V})$ & 0.25 & 0.2 & 0.3 & 0.4 & 0.4 & 0.3 \\
Bandwidth $(\mathrm{Hz})$ & $10 \mathrm{k}$ & $20 \mathrm{k}$ & $20 \mathrm{k}$ & $10 \mathrm{k}$ & $20 \mathrm{k}$ & $1 \mathrm{k}$ \\
Power consumption $(\mu \mathrm{W})$ & 7.5 & 7.5 & 79.3 & 0.41 & 140 & 0.85 \\
Sampling frequency $(\mathrm{Hz})$ & $1.4 \mathrm{M}$ & $12 \mathrm{M}$ & $2.56 \mathrm{M}$ & $2 \mathrm{M}$ & $3.2 \mathrm{M}$ & $256 \mathrm{k}$ \\
SNDR (dB) & 61 & 60.3 & 74 & 58 & 68 & 60.7 \\
DR (dB) & 64 & 68.9 & 77 & 64 & 74 & 65 \\
Technology (nm) & 130 & 90 & 130 & 130 & 130 & 65 \\
FOM $_{1}(\mathrm{pJ} / \mathrm{step})$ & 0.477 & 0.222 & 0.481 & 0.032 & 1.7 & 0.464 \\
$\mathrm{FOM}_{2}(\mathrm{~dB})$ & 155 & 163 & 161 & 168 & 155.5 & 156 \\
$\mathrm{FOM}_{3}(\mathrm{pJ} . \mathrm{V} / \mathrm{step})$ & 0.119 & 0.044 & 0.144 & 0.013 & 0.68 & 0.139 \\
\hline
\end{tabular}

${ }^{\dagger}$ Results from simulation. Other references provide experimental results.

The FOM 1 favors high-resolution ADCs, whereas the $\mathrm{FOM}_{2}$ favors high-DR ADCs. These FOM definitions disregard $V_{D D}$, the threshold voltage of the corresponding technology, and the available swing. According to the FOM definitions given above, the designed inverter-based, second-order modulator achieves $0.46 \mathrm{pJ} /$ conversion-step and $156 \mathrm{~dB}$, respectively, which are comparable to the other state-of-the-art modulators operating with supply voltages below $0.4 \mathrm{~V}$. Among others, the passive modulator in [2] operating at a $0.5 \mathrm{~V}$ supply consumes the lowest power (only $250 \mathrm{nW}$ ) while gaining moderate resolution ( $65 \mathrm{~dB}$ SNDR) and $72 \mathrm{~dB}$ DR. It looks attractive when using both FOM definitions given by Equations (12) and (13).

The modulator design in the ultra-low voltage domain (below $0.5 \mathrm{~V}$ ) is becoming more and more challenging due to the limited available signal swing and switch overdrive voltage. The output swing 
of an OTA directly determines the integrators' swing, which indeed defines the modulator reference voltage. As a consequence, the maximum input signal, $V_{\text {in.max }}$, that determines the DR of the ADC at a given input-referred noise floor $P_{n}$ is limited by the reference voltage or the integrators' swing. The DR can be derived as:

$$
D R=10 \log \frac{V_{\text {in.max }}^{2} / 2}{P_{n}+P_{D}}
$$

where $P_{D}$ is the input-referred distortion. To maintain the same DR in lower supply voltages, the noise floor and distortion power have to be decreased, requiring higher power consumption [21]. Generally speaking, for a better power efficiency, it is not desirable to reduce the supply voltage, because the analog power increases. However, specific applications, such as body implants (e.g., cardiac pacemakers, cochlear implants, etc.), demand ultra-low-voltage operation in the order of human body potentials.

It should be noted that the threshold voltage does not scale at the same proportion as the supply voltage, which limits the available overdrive voltage of the operating switches in SC designs. For fair comparisons of the modulators listed in Table 4, in addition to the commonly-used $\mathrm{FOM}_{1}$ and $\mathrm{FOM}_{2}$, the proposed $\mathrm{FOM}_{3}$ takes into account the $V_{D D}$, as well.

$$
F O M_{3}=\frac{\text { Power } \times V_{D D}}{2^{(S N D R-1.76) / 6.02} \times 2 \times B W}
$$

In fact, the most effective comparison among different modulators is when we consider the threshold voltage in addition to the supply voltage, $V_{D D}$, because the available voltage headroom for analog blocks and the overdrive voltage for the switching transistors (i.e., $V_{G S}-V_{T H}$ ) depend directly on $V_{T H}$. Therefore, for a fair comparison, we need to consider the term $V_{D D}-V_{T H}$ in the numerator of the $\mathrm{FOM}_{3}$ given by Equation (15), rather than merely $V_{D D}$. For simplicity, we assume all technologies used in Table 4 have the same $V_{T H}$. According to $\mathrm{FOM}_{3}$ and considering operating $V_{D D}$, the proposed modulator achieves a competitive figure of merit of $0.139 \mathrm{pJ} . \mathrm{V} /$ conversion-step.

\section{Conclusions}

An ultra-low-voltage, energy-efficient $\Delta \Sigma$ modulator is introduced in this paper for medical implant devices. In the absence of cascoding, a new gain-enhanced inverter-based amplifier was proposed to compensate for the reduced inverter's DC gain and GBW. To overcome the switches' overdrive limitation, the charge pump clock doublers were used to boost the clock signals' level. The single-bit quantizer, including the preamplifier, dynamic comparator and latch, was designed in the deep sub-threshold regime, providing very high power efficiency. The entire modulator operates reliably against PVT variations from a $300 \mathrm{mV} \pm 10 \%$ supply voltage. The modulator design is compatible with human body potentials, as well as the energy efficiency intended for the target applications.

Acknowledgments: The author would like to thank Gabor C. Temes of Oregon State University for his valuable guidelines, recommendations and proofreading. He also would like to thank Atila Alvandpour of Linköping University for the project support.

Author Contributions: In this research work, the author Ali Fazli Yeknami contributed to the literature search and came up with the idea of the new gain-enhanced inverter-based amplifier technique that can reliably work at supply voltages near $300 \mathrm{mV}$. He was responsible for simulating the entire delta-sigma modulator against PVT variations, for a comprehensive comparison with other ultra-low voltage designs. The manuscript was written by Ali Fazli Yeknami and proofread for the technical writing and formatting aspects by Gabor C. Temes of Oregon State University. The author also had extensive discussions with Atila Alvandpour at Linköping University from the standpoint of the project specifications and biomedical design requirements.

Conflicts of Interest: The author declares no conflict of interest. 


\section{References}

1. Muller, R.; Gambini, S.; Rabaey, J.M. A $0.013 \mathrm{~mm}^{2} 5 \mu \mathrm{W}$ DC-coupled neural signal acquisition IC with $0.5 \mathrm{~V}$ supply. IEEE J. Solid-State Circuits 2012, 47, 232-243. [CrossRef]

2. Yeknami, A.F.; Alvandpour, A. A 0.5-V 250-nW 65-dB SNDR Passive $\Delta \Sigma$ Modulator for Medical Implant Devices. In Proceedings of 2013 IEEE International Symposium on Circuits and Systems (ISCAS), Beijing, China, 19-23 May 2013; pp. 2010-2013.

3. Gerosa, A.; Neviani, A. A $1.8 \mu \mathrm{W}$ sigma-delta modulator for 8-bit digitization of cardiac signals in implantable pacemakers operating down to 1.8 V. IEEE Trans. Circuits Syst. II Express Briefs 2005, 52, 71-76. [CrossRef]

4. Zou, X.; Xu, X.; Yao, L.; Lian, Y. A 1-V 450-nW Fully Integrated Programmable Biomedical Sensor Interface Chip. IEEE J. Solid-State Circuits 2009, 44, 1067-1077. [CrossRef]

5. Lu, T.-C.; Van, L.-D.; Lin, C.-S.; Huang, C.-M. A 0.5 V 1 KS/s 2.5 nW 8.52-ENOB 6.8 fJ/Conversion-Step SAR ADC for Biomedical Applications. In Proceedings of IEEE Custom Integrated Circuits Conference (CICC), San Jose, SC, USA, 19-21 September 2011; pp. 1-4.

6. Yeknami, A.F.; Alvandpour, A. Low-Power Low-Voltage $\Delta \Sigma$ Modulator Using Switched-Capacitor Passive Filter. In VLSI-SOC: At the Crossroads of Emerging Trends; Orailoglu, A., Ugurdag, H.F., Sliveira, L.M., Margala, M., Reis, R., Eds.; Springer: Dordrecht, The Netherlands, 2015; Volume 461, pp. 94-118.

7. Yeknami, A.F.; Alvandpour, A. A 0.7-V 400-nW Fourth-Order Active-Passive Delta-Sigma Modulator with One Active Stage. In Proceedings of 21st IEEE International Conference on Very Large Scale Integration (VLSI-SoC), Istanbul, Turkey, 7-9 October 2013; pp. 1-6.

8. Yeknami, A.F.; Alvandpour, A. A $2.1 \mu \mathrm{W} 80 \mathrm{~dB}$ SNR DT $\Delta \Sigma$ modulator for medical implant devices in $65 \mathrm{~nm}$ CMOS. J. Analog Integr. Circuits Signal Proc. 2013, 77, 69-78. [CrossRef]

9. De Melo, J.L.; Querido, F.; Paulino, N.; Goes, J. A 0.4-V 410-nW Opamp-Less Continuous-Time $\Sigma \Delta$ Modulator for Biomedical Applications. In Proceedings of 2014 IEEE International Symposium on Circuits and Systems (ISCAS), Melbourne, Australia, 1-5 June 2014; pp. 1340-1343.

10. Roh, H.; Lee, H.; Choi, Y. A 0.8-V 816-nW delta-sigma modulator for low-power biomedical applications. J. Analog Integr. Circuits Signal Proc. 2010, 63, 101-106. [CrossRef]

11. Yeknami, A.F.; Qazi, F.; Alvandpour, A. Low-power DT $\Delta \Sigma$ modulators using SC passive filters in $65 \mathrm{~nm}$ CMOS. IEEE Trans. Circuits Syst. I 2014, 61, 358-370. [CrossRef]

12. Chae, Y.; Han, G. Low voltage, low power, inverter-based switched-capacitor delta-sigma modulator. IEEE J. Solid-State Circuits 2009, 44, 458-472. [CrossRef]

13. Pan, F.; Samaddar, T. Charge Pump Circuit Design, 1st ed.; McGraw-Hill Electronic Engineering Series; McGraw-Hill: New York, NY, USA, 2006.

14. Pun, K.P.; Chatterjee, S.; Kinget, P.R. A 0.5 V 74-dB SNDR 25-kHz continuous-time delta-sigma modulator with a return-to-open DAC. IEEE J. Solid-State Circuits 2007, 42, 496-507. [CrossRef]

15. Michel, F.; Steyaert, M. A $250 \mathrm{mV} 7.5 \mu \mathrm{W} 61 \mathrm{~dB}$ SNDR SC $\Delta \Sigma$ modulator using near-threshold-voltage-biased inverter amplifiers in $130 \mathrm{~nm}$ CMOS. IEEE J. Solid-State Circuits 2012, 47, 709-721. [CrossRef]

16. Enz, C.C.; Temes, G.C. Circuit techniques for reducing the effects of op-amp imperfections: autozeroing, correlated double sampling, and chopper stabilization. Proc. IEEE 1996, 84, 1584-1614. [CrossRef]

17. Yao, L.; Steyaert, M.; Sansen, W. Low-Power Low-Voltage Sigma-Delta Modulators in Nanometer CMOS; Springer: Dordrecht, The Netherlands, 2006.

18. Wismar, U.; Wisland, D.; Andreani, P. A 0.2 V, $7.5 \mu \mathrm{W}, 20 \mathrm{kHz} \Sigma \Delta$ Modulator with $69 \mathrm{~dB}$ SNR in $90 \mathrm{~nm}$ CMOS. In Proceedings of 33rd European Solid State Circuits Conference (ESSCIRC), Munich, Germany, 11-13 September 2007; pp. 206-209.

19. Qiao, Z.; Zhou, X.; Li, Q. A 250 mV 77 dB DR 10 kHz BW SC $\Delta \Sigma$ Modulator Exploiting Sub Threshold OTAs. In Proceedings of 40th European Solid State Circuits Conference (ESSCIRC), Venice, Italy, 22-26 September 2014; pp. 419-422. 
20. Yoon, Y.; Roh, H.; Roh, J. A True 0.4-V Delta-Sigma Modulator Using a Mixed DDA Integrator Without Clock Boosted Switches. IEEE Trans. Circuits Syst. II Express Briefs 2014, 61, 229-233. [CrossRef]

21. Yeknami, A.F. Low-Power Delta-Sigma Modulators for Medical Applications. Ph.D. Thesis, Linköping University, Linköping, Sweden, 21 January 2014.

(c) 2016 by the author; licensee MDPI, Basel, Switzerland. This article is an open access article distributed under the terms and conditions of the Creative Commons by Attribution (CC-BY) license (http:/ / creativecommons.org/licenses/by/4.0/). 